\title{
RETRACTED ARTICLE: 67-kDa laminin receptor induces FasL expression and FasL-mediated apoptosis through the activation of c-Myc and the subsequent activation of the FasL promoter in human cholangiocarcinoma cells
}

\author{
Shigang Duan · Dajiang Li • Zhanfeng Gao • \\ Jin Zhu $\cdot$ Yan Xiong · Long Chen · Xiaowu Li · Shuguang Wang
}

Received: 7 July 2009 / Accepted: 28 January 2010 / Published online: 13 February 2010

(c) Springer-Verlag 2011

This article has been withdrawn due a duplicated submission/publication of the work published in Digestive Diseases and Sciences.

S. Duan $\cdot$ D. Li $\cdot$ Z. Gao $\cdot$ J. Zhu $\cdot$ Y. Xiong $\cdot$ L. Chen $\cdot$ X. Li $\cdot$

S. Wang $(\bowtie)$

Hepatobiliary Surgery Hospital and Institute,

Southwest Hospital, Third Military Medical University,

Chongqing 400038, China

e-mail: sgwang90@yahoo.com 